\title{
Relevance of Leaflet Prolapse to the Indication Policy for Aortic Valve- Sparing Root Replacement
}

\author{
Andreas Borowski, MD, ${ }^{1}$ Jan-Philipp Minol, MD, ${ }^{1}$ Patric Kröpil, MD, ${ }^{2}$ Philipp Rellecke, MD,${ }^{1}$ \\ Arash Hehdiani, MD, ${ }^{1}$ Hannan Dalyanoglu, $\mathrm{MD}^{1}$ \\ ${ }^{1}$ Departments of Cardiovascular Surgery and ${ }^{2}$ Radiology, University Hospital, Dusseldorf, Germany
}

\section{ABSTRACT}

Background: In aortic root replacement, "preexisting" or "induced" aortic leaflet prolapse is related to advanced aortic root pathology and can indicate valve repair. Efforts should be made to perform root replacement before leaflet prolapse is in its maximum extent.

Materials and Methods: Thirty-nine patients with chronic aortic root dilatation and aortic valve regurgitation (AR) underwent a reimplantation procedure. Contrary to 32 of the 39 patients (group A), 7 of the 39 patients (group B) underwent cusp plication for prolapse. For both groups, data related to the diameter at the level of maximal tubular extension, sinotubular junction, sinus of Valsalva, aortoventricular junction (AVJ), and aortic annulus were obtained from preoperative computed tomography scans and analyzed comparatively.

Results: Group B showed a higher mean AR grade $(P=.007)$, a higher mean diameter at the level of the aortic annulus $(P=.038)$, AVJ $(P=.037)$, and aortic sinus $(P<.001)$ and a higher sinus dilatation index (existing-to-predicted diameter ratio) $(P<.001)$ than group A. The sinus of Valsalva displayed the best predictive value regarding a plicatureindicating prolapse $(P<.001 ; 95 \%$ confidence interval $[\mathrm{CI}]$ : $0.809-1.013)$. A diameter $>40 \mathrm{~mm}$ was accompanied by an odds ratio (OR) of 24.6 (95\% CI: 1.29-496.02).

During the follow-up period of $29.0 \pm 18.4$ months (range: 6-62 months), 1 patient (group A) required reoperation 5 years postoperatively for progressive AR.

Conclusion: The sinus of Valsalva diameter seems to have the greatest prognostic value for the development of prolapse.

Received September 9, 2018; accepted Fanuary 2, 2019.

A.B. and 7.-P.M are co-first authors of the article because they contributed equally to this work.

Abbreviations: AR, aortic valve regurgitation; $A U C$, area under the curve; $A V 7$, aorto-ventricular junction; BSA, body surface area; CC, cross-clamp of the aorta; $C P B$, cardiopulmonary bypass; $C T$, computed tomography; IDDM, insulin-dependent diabetes mellitus; OR, odds ratio; ROC, receiver operating characteristic; STF, sinotubular junction; SD, standard deviation; TEE, transesophageal echocardiography.

Correspondence: Jan-Philipp Minol, MD, Department of Cardiovascular Surgery, University Hospital, Moorenstr. 5, 40225 Duesseldorf, Germany; +49-(0)21181-07636; fax: +49-(0)211-81-18-333 (e-mail: phil.minol@gmx.de).
Our data suggest that root repair should be considered earlier in time before leaflet prolapse is complete, which most likely occurs when root dilatation becomes an aneurysm.

\section{INTRODUCTION}

After valve-preserving aortic root repair surgery for chronic aortic root aneurysms, the pre- and postrepair cusp configurations are known to influence the long-term outcome [Oka 2011; David 2014; Stephens 2014]. The size of the aortic root has been reported to be a significant predictor of late aortic valve regurgitation (AR) and its possible progression, thus leading to the need for reoperation and valve replacement [Kunihara 2012].

Leaflet dimensions change in parallel with aortic dilatation, resulting in cusp prolapse [Thubrikar 2005]. As an underlying mechanism, an adaptation process occurs with leaflet enlargement, and cusp prolapse develops as a result of the limited adaptation capability [Yacoub 1999; Chester 2000; Kim 2014]. This type of prolapse can be identified preoperatively (so-called "preexisting" prolapse). However, a leaflet that is enlarged as a result of the adaptation process may effectively prevent $A R$ and remain undetected. Surgical intervention by inserting a tubular graft can, per se or by a technical error, aggravate this pathology and induce iatrogenic prolapse (socalled "induced" prolapse) [Schäfers 2013].

Because the likelihood of AR originating from preexisting or induced prolapse increases with increasing root diameter [Padial 1997], it is of interest to delineate the most predictive parameter in the geometry of the aortic root regarding a cusp prolapse, thus optimizing the timing for prophylactic surgical intervention. In this context, a threshold diameter indicating the need for surgery in patients with AR would be desirable.

\section{MATERIALS AND METHODS}

Between 2010 and 2015, 184 patients underwent the David procedure for ascending aneurysms. Of these, 39 consecutive patients with $\mathrm{AR}$ and underlying root pathology were included in this study. The inclusion criteria were chronic root dilatation with a regurgitant tricuspid aortic valve with any degree of regurgitation and repair by a valve-sparing root replacement using the reimplantation technique described by Tirone David, referred to as the David procedure [David 


\section{Intergroup Comparison of Sinus}

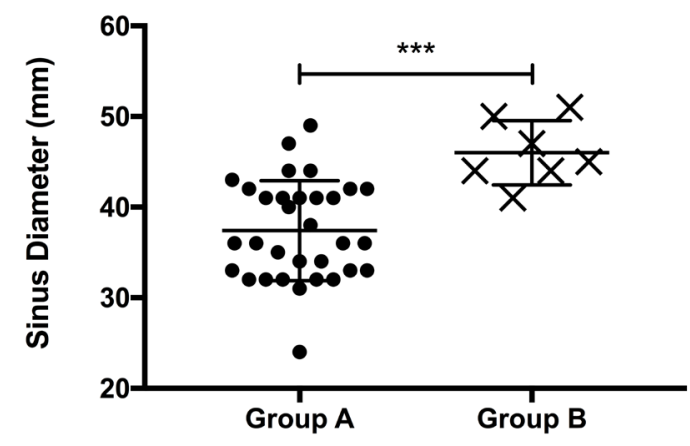

Direct comparison of the sinus diameter in both groups. Mann-Whitney $U$ test: $* * * P<.001$.

1992]. Aortic dilatation was defined as an aortic diameter exceeding the normal value predicted for the patient using age and body surface area (BSA) according to Roman's formula [Roman 1989]; dilation was considered mild if the aortic ratio was 1.1-1.25, moderate if the ratio was 1.26-1.49, and severe if the ratio was $>1.5$ at any of the 3 measurement levels (sinus of Valsalva, sinotubular junction [STJ], or mid-ascending aorta).

As in all of our patients, AR was associated with aortic dilatation; the level of the lesion(s) was recorded in each individual patient according to the AR classification as described by Boodhvani [Boodhwani 2009]: type Ia (tubular and STJ enlargement), type Ib (sinus and STJ enlargement), type Ic (AVJ enlargement), type II (prolapse).

Patients with acute aortic dissection, a bicuspid aortic valve, connective tissue disorders (eg, Ehlers-Danlos type IV, Marfan, or Loeys-Dietz syndrome), or a familial history of aneurysm dissection were excluded from the study.

AR was assessed using transesophageal echocardiography (TEE), and the degree of AR was graded semiquantitatively on a scale from $1+$ to $3+$, based on the jet width and standard criteria.

Aortic valve function was routinely assessed postoperatively during the predischarge examination and after discharge by an experienced cardiographer in an outpatient setting.

All patients were operated on by using cardiopulmonary bypass $(\mathrm{CPB})$ and cold blood cardioplegia for myocardial protection. The choice of the graft tube size was based on the annulus measurements and by consideration of the BSA, according to Schäfers' formula [Schäfers 2015b].

Leaflet prolapse was corrected by central plication of the free margin using 6-0 polypropylene sutures (Prolene, Ethicon, Inc., Hamburg, Germany) in all cases, except one, immediately after completion of the root replacement or, as in one case, following TEE examination, which was performed routinely after the discontinuation of CPB.

Aortic diameter measurements were performed in all patients at the level of the maximal tubular extension $(a)$, STJ $(b)$, aortic sinus (c), aorto-ventricular junction (AVJ) (d), and aortic annulus (e) by using computed tomography (CT). All measurements were obtained from images perpendicular to the centerline of the vessel from reconstructed CT scans.
Table 1. Demographic and Clinical Data* $†$

\begin{tabular}{lccc}
\hline & $\begin{array}{c}\text { Group A } \\
(\mathrm{n}=32)\end{array}$ & $\begin{array}{c}\text { Group B } \\
(\mathrm{n}=7)\end{array}$ & $P$ \\
\hline Age (y) & $70.6 \pm 7.8$ & $58.8 \pm 8.6$ & $<.001$ \\
Female & $21(65.6 \%)$ & $3(42.9 \%)$ & .394 \\
BSA (m $\left.{ }^{2}\right)$ & $1.89 \pm 0.2$ & $1.97 \pm 0.2$ & .662 \\
Hypertension & $19(59.4 \%)$ & $6(85.7 \%)$ & .002 \\
IDDM & $3(9.4 \%)$ & $0(0 \%)$ & 1 \\
AR preop (grade) & $2.0(1-3)$ & $3.0(2-3)$ & .007 \\
$\quad$ Grade I & $4(12.5 \%)$ & $0(0 \%)$ & \\
Grade II & $20(62.5 \%)$ & $2(28.6 \%)$ & \\
Grade III & $8(25.0 \%)$ & $5(71.4 \%)$ & \\
Dacron tube diameter (mm) & $28(26-30)$ & $28(24-30)$ & .617 \\
CC time (min) & $123.2 \pm 26.2$ & $147.2 \pm 4$ & .2 \\
CPB time (min) & $160.5 \pm 36.2$ & $197.7 \pm 46.4$ & .082 \\
AR postop (grade) & $0(0-2)$ & $0(0-1)$ & .464 \\
Grade I & $9(28.2 \%)$ & $1(14.3 \%)$ & \\
Grade II & $1(3.2 \%)$ & $0(0 \%)$ & \\
Grade III & $0(0 \%)$ & $0(0 \%)$ & \\
\hline
\end{tabular}

*Preoperative patient characteristics and perioperative data. Categorical variables are expressed as proportions. Continuous variables are generally given as the mean \pm standard deviation (SD). The Dacron tube size and aortic regurgitation (AR) grade are exclusively given as the median and range. BSA, body surface area; IDDM, insulin-dependent diabetes mellitus; AR, aortic valve regurgitation; $\mathrm{CC}$, cross-clamp of the aorta; $\mathrm{CPB}$, cardiopulmonary bypass.

Normal values for the aortic root diameter were calculated and adjusted for age and sex for each individual patient according to the Framingham Heart Study [Dawber 1951; Vasan 1995]. BSA was calculated by using the Du Bois formula [Du Bois 1916].

The degree of aortic root dilatation at the sinus level is expressed by the aortic dilatation index, which corresponded to the existing-to-predicted diameter ratio.

All patients were divided into 2 groups. Those with no concomitant cusp repair were allocated to group A, and those in whom cusp plication for prolapse was performed were allocated to group B. The preoperative characteristics, perioperative course, and postoperative outcome were compared between these 2 groups.

The predictive value of the above-mentioned parameters a to e regarding a leaflet prolapse requiring plication was examined by receiver operating characteristic (ROC) curve analyses of the total group of 39 patients.

\section{Statistics}

For statistical analyses, the Mann-Whitney $\mathrm{U}$ test and Fisher exact test were used for continuous and categorical variables, respectively. Differences were considered significant at $P<.05$. 
Table 2. Preoperative Aortic Measurements*

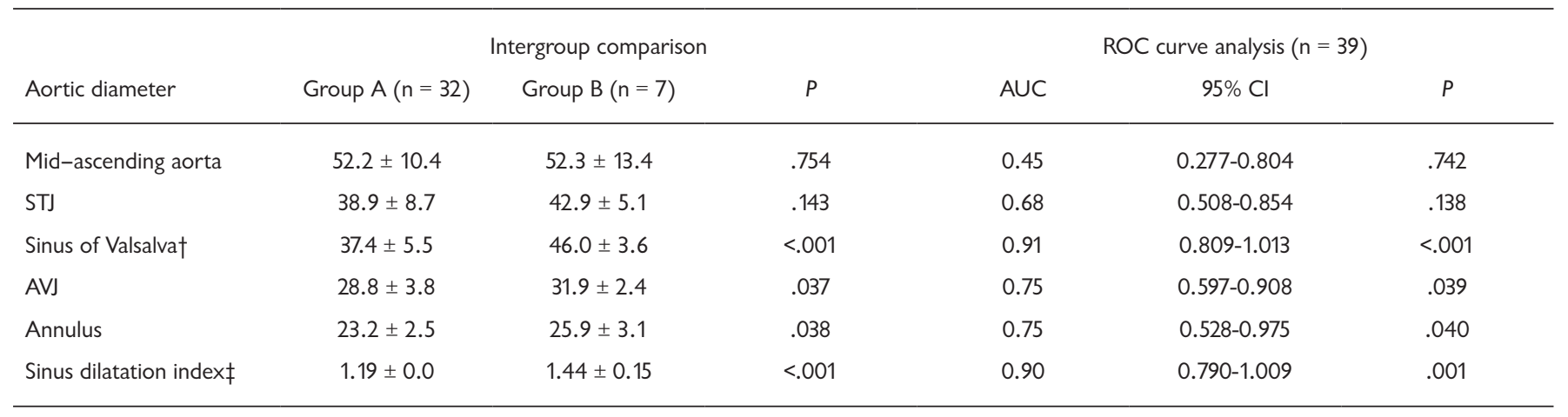

*Preoperative measurements of the aorta are displayed as the mean \pm standard deviation. STJ, sinotubular junction; AVJ, aorto-ventricular junction. $\dagger$ The ROC curve analysis revealed the best predictive value for the sinus of Valsalva diameter regarding a plication-indication prolapse (AUC 0.91; $P<.001 ; 95 \%$ Cl: 0.809-1.013).

$\ddagger$ According to the existing-to-predicted diameter ratio-for explanation, see "Materials Methods." ROC curve analysis revealed the sinus of Valsalva as the best predictive parameter regarding a plicature-indicating prolapse $(P<.001)$ next to the sinus dilatation index $(P=.001)$.

For the above-mentioned parameters a to e, ROC curves were established regarding the finding of a plicationindicating prolapse as described elsewhere [Green 1966; Metz 1978; Swets 1979; Hanley 1982]. For each ROC curve, the area under the curve (AUC) and its relation to the null hypothesis were analyzed. Finally, we calculated the odds ratio $(\mathrm{OR})$ of an aortic sinus diameter $>40 \mathrm{~mm}$ regarding the finding of a plication-indicating prolapse (Prism 7, GraphPad Software, La Jolla, CA, USA).

\section{RESULTS}

The demographic characteristics and distributions of age, sex, BSA, comorbidities, surgical data (eg, Dacron tube size, aortic cross-clamp time, CPB time), pre- and postoperative AR, and the degree of aortic dilatation with calculated ratios are summarized in Table 1. Patients in group B had a significantly higher degree of preoperative AR $(P<.007)$.

All but 2 patients (from group B) fulfilled the root dilatation criteria, and the majority of patients had a mild degree of dilatation. In the remaining 2 patients in group B, the aortic root diameter reached the criteria for aneurysm.

In group A, 10 patients had 1 lesion (aortic dilatation at 1 level only) (8 patients, type Ia; 2 patients, type Ic), 15 patients had 2 lesions (14 patients, types Ia and Ib; 1 patient, types Ib and Ic), and 7 patients had 3 lesions (all, types Ia, Ib, and Ic). In group B, 1 patient had 1 lesion (type Ib), 2 patients had 2 lesions (both, types Ia and Ib), and 4 patients had 3 lesions (all, types Ia, Ib, and Ic).

In group B, 2 patients had preexisting prolapse, 4 patients had induced prolapse that was identified after insertion of the tube graft, and 1 patient had induced prolapse that was detected after CPB discontinuation.

Table 2 presents the aortic diameter measured at the level of the mid-ascending segment, STJ, sinus of Valsalva, AVJ, aortic annulus, and sinus dilatation index for groups $\mathrm{A}$ and $\mathrm{B}$ and the ROC curve analysis results. Group B showed a significantly greater diameter at the level of the aortic sinus, AVJ, and aortic annulus, as well as a significantly greater sinus dilatation index.

A comparative presentation of the sinus of Valsalva of both groups is given in the Figure. In addition to the intraand intergroup characteristics $(P<.001)$ given in Table 2 , it emphasizes the finding that no prolapse was found in any patient with a sinus diameter $\leq 40 \mathrm{~mm}$. The reverse consideration showed an OR of 24.6 (95\% CI: 1.290-469.022; $P=.033)$ for a sinus of Valsalva diameter $>40 \mathrm{~mm}$ regarding a plication-indicating prolapse, which is approximately 1.3 -fold larger than the expected size for our patient cohort.

No hospital deaths were observed. At the predischarge TEE examination, 10 patients in group A and 1 patient in group B exhibited grade 1+ to 2+ AR. Clinical follow-up for all patients ranged between 6 and 62 months, with an average of $29 \pm 18.4$ months. During the follow-up time, 1 patient (group A) died from noncardiac causes. No progression of AR was observed in any but 1 patient from group A, who developed progressive $\mathrm{AR}$ and required a repeat surgery with aortic valve replacement 5 years postoperatively.

\section{DISCUSSION}

By common convention, an aortic aneurysm is defined as a local increase in the diameter of more than $50 \%$ of that predicted for a patient's age, sex, and body size. Diameters less than $50 \%$ of the normal aortic diameter are defined as ectasia. An official cutoff diameter for the definition of dilatation has not been determined because of the lack of a simple measurement method, which has led to low practicability [Saliba 2015].

Although the natural history of aortic aneurysm is characterized by indolent growth, alterations in the root geometry can have deleterious effects on the aortic valve leaflets 
by changing their dimensions, eventually resulting in $\mathrm{AR}$ [Thubrikar 2005].

Typical changes in leaflet dimensions are related to increased free-edge length, leaflet height, and surface area, whereas enlargement of the aortic annulus does not necessarily follow an increase in the root diameter.

Thubrikar et al [Thubrikar 2005] reported that in 3 of 14 patients with root dilatation and AR grades of 3+ and 4+, the annulus diameter was less than $23.5 \mathrm{~mm}$ and the STJ-toannulus ratio was between 1.2 and 1.6. The authors, unfortunately, disregarded the sinus of Valsalva diameter, focused only on the STJ, and did not report any correlation between the STJ diameter and the degree of AR. They suggested that the development of AR depended on multiple factors.

Della Corte et al [Della Corte 2006] reported that of 301 patients with mild-to-severe AR and underlying aortic dilatation, $76 \%$ had a mean sinus diameter of $3.8 \pm 0.6 \mathrm{~cm}$.

Analysis of data reported by cardiac surgery centers with regard to root diameter and degree of AR at surgery revealed that surgery is usually performed on patients with very advanced leaflet pathology and associated AR, despite mild root dilatation.

A very interesting contribution to understanding the mechanism of AR was recently published by Kim et al [Kim 2014]. The authors compared the root diameter in 2 patient cohorts, with and without AR, in relation to the normal aortic diameter. They found that during the course of root dilatation, leaflets adapted by increasing their surface area, but the aortic valve could remain competent even if the surface area was augmented by more than $50 \%$ of the baseline value. Grade 3+ and 4+ AR levels were observed as early as the surface area was augmented by $100 \%$ or more.

Based on our measurements and suggestions and those of Thubrikar et al [Thubrikar 2005], if the diameter of the sinus of Valsalva plays the most important role in AR development, then an individual approach with geometry adaptations and either 1-component (root-only) or 2-component (root-andleaflet) surgery seems to be logical and justified.

Schäfers, who generally prefers the remodeling technique, replaces aortic sinuses only when the AVJ diameter does not exceed $29 \mathrm{~mm}$ and the maximal sinus diameter is larger than $45 \mathrm{~mm}$; a more aggressive approach with reimplantation is performed for an AVJ diameter larger than $29 \mathrm{~mm}$.

Interestingly, root repair is not performed on Schäfers' patients when the sinus diameter is less than $45 \mathrm{~mm}$, which is generally in accordance with the 2008 and 2014 ACC/AHA guidelines for aortic root dilatation treatment [Cozijnsen 2011; Erbel 2014]. At Schäfers' institution, cusp repair is performed when the geometric height of the cusp exceeds 18-19 $\mathrm{mm}$ [Schäfers 2013]; the tubular graft is chosen according to the BSA of the patient: $24 \mathrm{~mm}$ for a BSA $<1.8 \mathrm{~m}^{2}, 26 \mathrm{~mm}$ for $1.9-2.2 \mathrm{~m}^{2}$, and $28 \mathrm{~mm}$ for $>2.3 \mathrm{~m}^{2}$ [Schäfers 2015b].

According to data reported from very experienced largevolume centers [Della Corte 2006; Schäfers 2015a], at the time of surgery, more than $75 \%$ of patients with root dilatation had AR preoperatively, and in $50 \%-75 \%$ of patients, a 2-component repair including concomitant cusp plication was performed. The estimated percentage of induced prolapse related to repair has not yet been reported.

Currently, the most intriguing issue regarding repair relates to the standardization of both, the remodeling and the reimplantation techniques, as well as to their reproducibility in terms of excellent results, especially in patients with AR and associated prolapse.

Despite successful attempts to standardize and reproduce the reimplantation technique, Schäfers' standards are not yet established, even in expert centers, and the same is true for reimplantation technique.

Although in experienced high-volume centers the remodeling or reimplantation technique reportedly provided excellent midterm results in terms of survival rates, freedom of recurrent $\mathrm{AR}$ and of reoperation, the incidence of postoperative $\mathrm{AR}$ is still frequent, especially when cusp repair was necessary.

Relevant studies from experienced centers provide evidence hereof and information about the prognostic aspects of postoperative AR in midterm follow-up:

Miyahara [Miyahara 2013] observed in a patient collective of 86 patients after aortic root replacement with and without cusp repair, after a median follow-up of 46 months, the development of moderate AR or greater in 14 patients (16\%).

Miller's group from Stanford [Stephens 2014] reported about 73 patients with mixed aortic pathology who underwent David procedure and cusp repair, of whom $43 \%$ were diagnosed 1-year postoperatively with mild $\mathrm{AR}$ and $5 \%$ were diagnosed with moderate $\mathrm{AR}$. In all patients with moderate $\mathrm{AR}$ and in $85 \%$ with mild $\mathrm{AR}$, they remained stable at 5 -year follow-up. Progression from mild-to-moderate AR occurred in $12 \%$ of the patients, and at a median follow-up of 28 months. One patient developed a severe AR and needed surgery.

El Khouri's group [El Khoury 2004] operated on 44 patients with prolapse (19 cases, David procedure) and observed at discharge a mild AR in 7 patients and a progression to a moderate AR in 4 of the 7 patients at a mean followup of 15 months.

Malvindi [Malvindi 2015] reported that in 139 patients after the David procedure (13\% cusp repair) a freedom from reoperation on the aortic valve at 1 year of $96 \%$, at 5 years of $90 \%$, and at 8 years of $86 \%$ for the whole patient collective, and a significantly higher rate of reoperation in patients who received leaflet repair.

Settepani [Settepani 2016] observed that in 157 patients after the David procedure (12\% cusp repair) a freedom from aortic valve reoperation of $96 \%$ at 1 year, of $92 \%$ at 5 years, and of $89 \%$ at 12 years, whereas reoperation rate was significantly higher in patients who received cusp repair compared to those who did not, with freedom from reoperation at 8 years of $58 \%$ and $94 \%$, respectively.

Tanaka [Tanaka 2017] published results of a multicenter study on 249 patients operated on with the David technique (among them 163 patients with cusp repair). Tanaka and colleagues observed freedom from greater-than-mild $\mathrm{AR}$ of $82 \%$ and $77 \%$ at 5 and 8 years, respectively, and freedom from aortic valve reoperation of $93 \%$ and $87 \%$ at 5 and 8 years, respectively, concluding that preoperative cusp prolapse was a risk factor, whereas cusp repair was not a risk factor for recurrent AR.

Esaki's [Esaki 2017] investigations showed that the 
cusp repair was an independent risk factor for late aortic valve dysfunction.

In summary, in patients who received aortic root surgery with cusp repair, a mild postoperative $\mathrm{AR}$ is frequent with an incidence of approximately $15 \%-40 \%$ within the first postoperative year. A progression from mild-to-moderate AR during the first postoperative year occurs in $15 \%-50 \%$ of the patients, whereas up to $23 \%$ of the patients can develop a more-than-mild AR at 8 years postoperatively. In the longterm, the freedom from reoperation on aortic valve at 8 years can reach values of as low as $58 \%$ (range $58 \%-87 \%$ ). According to Miller's statement, we need a 10-to-15-year follow-up to learn the long-term clinical course of postoperative mild AR. Preoperative cusp prolapse remains a risk factor for recurrent AR.

\section{Limitation of the Study}

The major drawback of the study is a short follow-up, which makes an assessment of durability of the cusp repair impossible.

\section{CONCLUSION}

The sinus diameter seems to have the greatest prognostic value for the development of prolapse. On the basis of our observations and those of others, AR with preexisting or induced prolapse can occur in a considerable number of patients with aortic sinus slightly larger than $40 \mathrm{~mm}$ in diameter.

Therefore, more robust and sophisticated preoperative diagnostic monitoring would be advantageous for the detection of the onset of leaflet prolapse. In patients with associated root pathology and AR, a lower sinus diameter threshold of 40-45 $\mathrm{mm}$ should be considered for surgery.

\section{REFERENCES}

Boodhwani M, de Kerchove L, Glineur D, et al. 2009. Repair-oriented classification of aortic insufficiency: impact on surgical techniques and clinical outcomes. J Thorac Cardiovasc Surg 2009;137:286-94.

Chester AH, Misfeld M, Yacoub MH. 2000. Receptor-mediated contraction of aortic valve leaflets. J Heart Valve Dis 9:250-4; discussion 254-5.

Cozijnsen L, Braam RL, Waalewijn RA, et al. 2011. What is new in dilatation of the ascending aorta? Review of current literature and practical advice for the cardiologist. Circulation 123:924-8.

David TE. 2014. Current readings: aortic valve-sparing operations. Semin Thorac Cardiovasc Surg 26:231-8.

David TE, Feindel CM. 1992. An aortic valve-sparing operation for patients with aortic incompetence and aneurysm of the ascending aorta. J Thorac Cardiovasc Surg 103:617-22; discussion 622.

Dawber TR, Meadors GF, Moore FE Jr. 1951. Epidemiological approaches to heart disease: the Framingham Study. Am J Public Health Nations Health 41:279-81.

Della Corte A, Romano G, Tizzano F, et al. 2006. Echocardiographic anatomy of ascending aorta dilatation: correlations with aortic valve morphology and function. Int J Cardiol 113:320-6.
Du Bois D, Du Bois EF. 1916. Clinical calorimetry: tenth paper[,] a formula to estimate the approximate surface area if height and weight be known. Arch Intern Med (Chic) 17:863-71

El Khoury G, Vanoverschelde JL, Glineur D, et al. 2004. Repair of aortic valve prolapse: experience with 44 patients. Eur J Cardiothorac Surg. 26:628-33.

Erbel R, Aboyans V, Boileau C, et al. 2014. 2014 ESC guidelines on the diagnosis and treatment of aortic diseases: document covering acute and chronic aortic diseases of the thoracic and abdominal aorta of the adult. The Task Force for the diagnosis and treatment of aortic diseases of the European Society of Cardiology (ESC). Eur Heart J 35:2873-926.

Esaki J, Leshnower BG, Binongo JN, et al. 2017. Risk factors for late aortic valve dysfunction after the David $\mathrm{V}$ valve-sparing root replacement. Ann Thorac Surg 104:1479-87.

Green DM, Swets JA. 1966. Signal detection Theory and Psychophysics. New York: Wiley.

Hanley JA, McNeil BJ. 1982. The meaning and use of the area under a receiver operating characteristic (ROC) curve. Radiology 143:29-36.

Kim DH, Handschumacher MD, Levine RA, et al. 2014. Aortic valve adaptation to aortic root dilatation: insights into the mechanism of functional aortic regurgitation from 3-dimensional cardiac computed tomography. Circ Cardiovasc Imaging 7:828-35.

Kunihara T, Aicher D, Rodionycheva S, et al. 2012. Preoperative aortic root geometry and postoperative cusp configuration primarily determine long-term outcome after valve-preserving aortic root repair. J Thorac Cardiovasc Surg 143:1389-95.

Malvindi PG, Cappai A, Basciu A, et al. 2015. David operation: single center 10-year experience. J Cardiovasc Surg (Torino) 56:639-45.

Metz CE. 1978. Basic principles of ROC analysis. Semin Nucl Med 8:283-98.

Miyahara S, Omura A, Sakamoto T, et al. 2013. Impact of postoperative cusp configuration on midterm durability after aortic root reimplantation. J Heart Valve Dis 22:509-16.

Oka T, Okita Y, Matsumori M, et al. 2011. Aortic regurgitation after valve-sparing aortic root replacement: modes of failure. Ann Thorac Surg 92:1639-44.

Padial LR, Oliver A, Sagie A, Weyman AE, King ME, Levine RA. 1997. Two-dimensional echocardiographic assessment of the progression of aortic root size in 127 patients with chronic aortic regurgitation: role of the supraaortic ridge and relation to the progression of the lesion. Am Heart J 134(5 Pt 1):814-21.

Roman MJ, Devereux RB, Kramer-Fox R, O'Loughlin J. 1989. Twodimensional echocardiographic aortic root dimensions in normal children and adults. Am J Cardiol 64:507-12.

Saliba E, Sia Y. 2015. The ascending aortic aneurysm: when to intervene? Dore A, El Hamamsy I, collaborators. Int J Cardiol Heart Vasc 6:91-100.

Schäfers HJ. 2015. Aortic valve repair: easy and reproducible? J Thorac Cardiovasc Surg 149:129-30.

Schäfers HJ, Aicher D. 2013. Root remodeling for aortic root dilatation. Ann Cardiothorac Surg 2:113-6.

Schäfers HJ, Raddatz A, Schmied W, et al. 2015. Reexamining remodeling. J Thorac Cardiovasc Surg 149(2 suppl):S30-6.

Settepani F, Cappai A, Basciu A, et al. 2016. Impact of cusp repair on reoperation risk after the David procedure. Ann Thorac Surg 102:1503-11. 
Stephens EH, Liang DH, Kvitting JP, et al. 2014. Incidence and progression of mild aortic regurgitation after Tirone David reimplantation valve-sparing aortic root replacement. J Thorac Cardiovasc Surg 147:169-77, 178.e1-178.e3.

Swets JA. 1979. ROC analysis applied to the evaluation of medical imaging techniques. Invest Radiol 14:109-21.

Tanaka H, Takahashi H, Inoue T, et al. 2017. Which technique of cusp repair is durable in reimplantation procedure? Eur J Cardiothorac Surg $52: 112-7$
Thubrikar MJ, Labrosse MR, Zehr KJ, Robicsek F, Gong GG, Fowler BL. 2005. Aortic root dilatation may alter the dimensions of the valve leaflets. Eur J Cardiothorac Surg 28:850-5.

Vasan RS, Larson MG, Levy D. 1995. Determinants of echocardiographic aortic root size. The Framingham Heart Study. Circulation 91:734-40.

Yacoub MH, Kilner PJ, Birks EJ, Misfeld M. 1999. The aortic outflow and root: a tale of dynamism and crosstalk. Ann Thorac Surg 68(3 suppl):S37-43. 